\title{
Artificial means for improving human motion
}

\author{
Diana Hodgins \\ European Technology for Business (ETB) Ltd, Herts, UK
}

\begin{abstract}
This paper gives a brief description of some of the key ongoing product developments in Europe for medical implants. A range of medical systems that are currently being developed under European-funded programmes are briefly described. The first is an implant that helps people with 'dropped foot' improve their walking. Further research on body-worn inertial measuring systems (IMUs) and microsystems will extend the capabilities to other lower-limb and upper-limb applications in the future. Other medical systems being developed with the use of microsystems and nanotechnology include a system to aid deaf and blind people.
\end{abstract}

Keywords: medical implant, microsystem, nanotechnology, European funding

Many people suffer from impaired limb movement due to either illness or accident. Conventional methods to improve movement include artificial aids such as splints attached to the body.

One example of reduced limb movement is with people with 'dropped foot'. This condition often occurs with stroke patients, where the person has difficulty with lifting the foot during the swing phase of gait. Conventional treatment is a splint attached to support the ankle joint. As a development of earlier systems, in the early 1990s, Salisbury Hospital developed a surface stimulator that pulses an electrical signal into the nerve from external electrodes carefully located on the lower leg. This stimulator, including power and signal conditioning circuitry, is triggered to send the pulse into the leg by a foot switch wired into the surface stimulator. This system offers some advantages when compared with the splint and is currently used by several thousand patients across Europe.

The team at Salisbury Hospital, together with RRD (a research group in Holland), end-user manufacturer Finetech Medical (FTM) and microsystem design house European Technology for Business (ETB), have extended this principle to produce an implantable functional electrical stimulator (FES) system for the dropped foot application. This system still has the external power unit and signal processing to produce an electrical pulse mounted on the calf. It also still has the foot switch to trigger the signal. The main difference between the implant and the surface stimulator is that the electrical pulse is fed directly to 2 branches of the common peroneal nerve. The implant comprises passive receive electronics, a cable to transmit the electrical pulse and 2 electrodes attached directly to the nerves (see Figure 1).
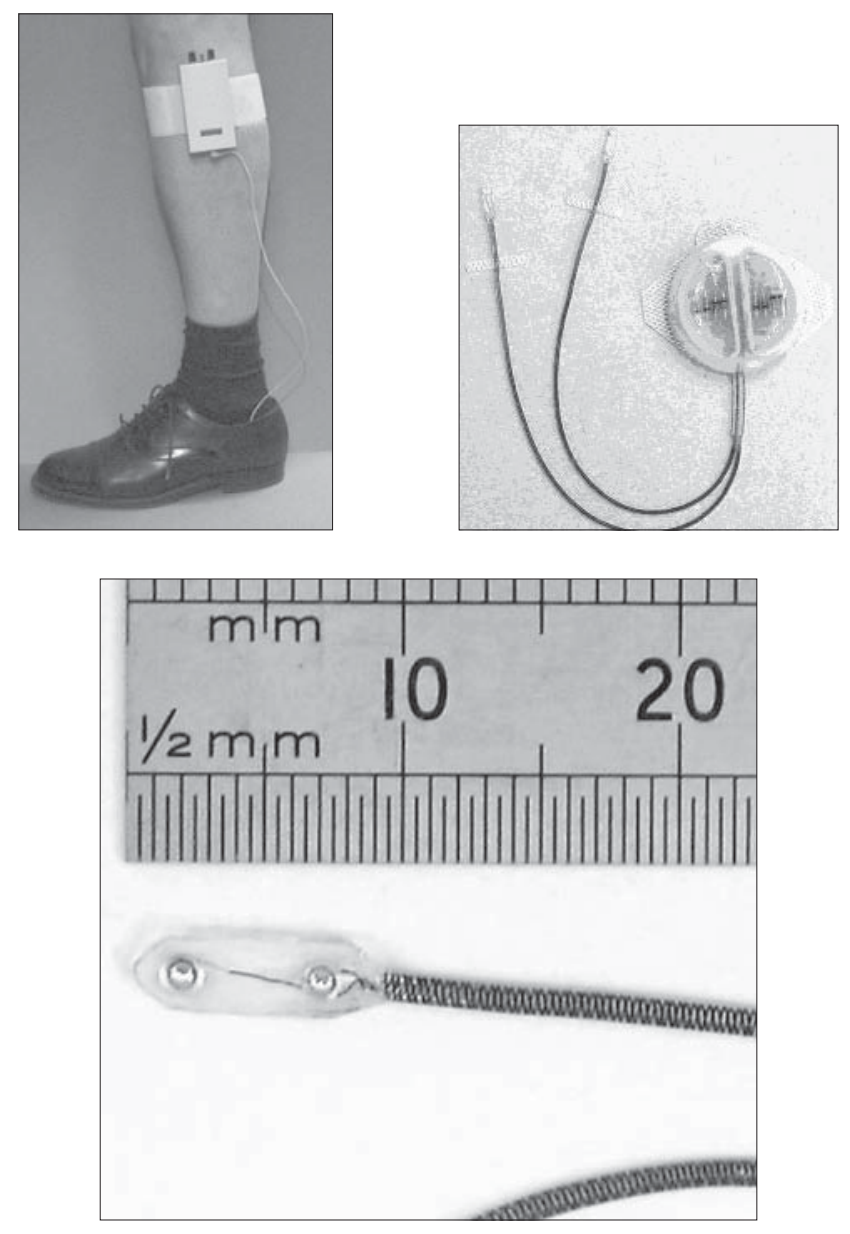

Figure I Various components of the 'dropped foot' implant system.

Correspondence: Diana Hodgins, European Technology for Business (ETB) Ltd, Codicote Innovation Centre, St Albans Road, Codicote, Herts SG4 8WH, UK; tel +44 I 438822 822; fax +44 I 438822 8I I; email diana.hodgins@etb.co.uk 

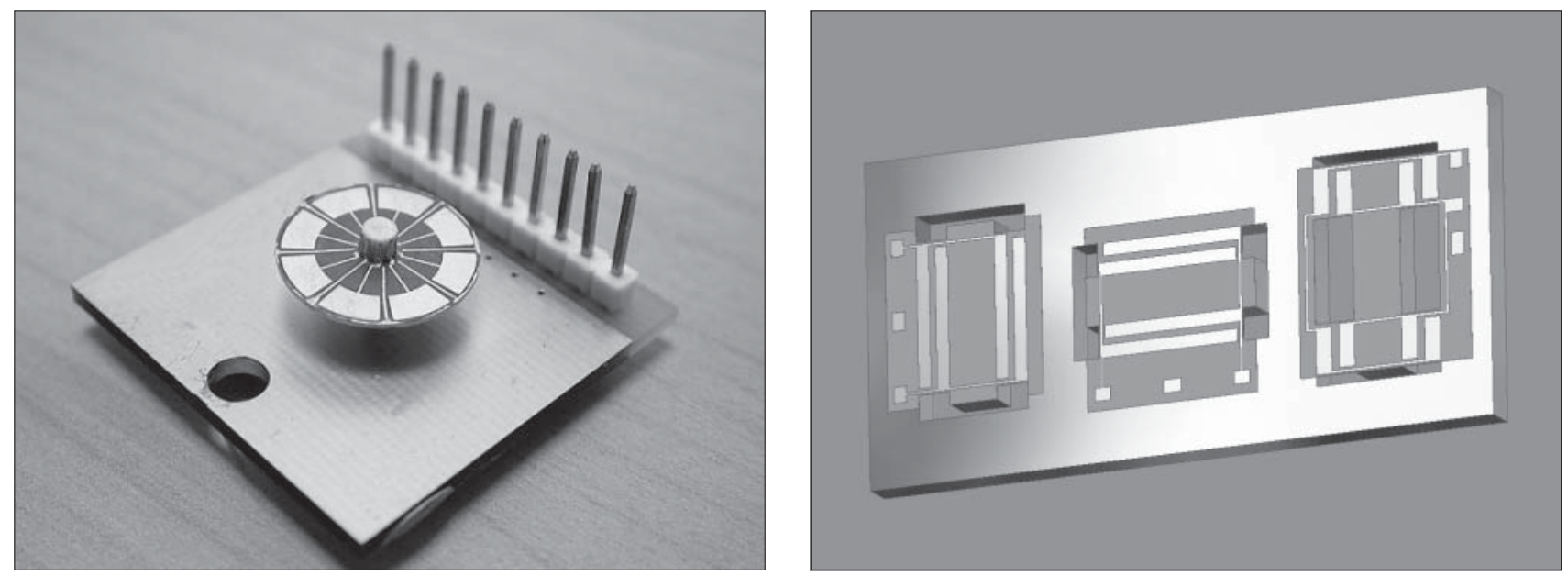

Figure 2 The ETB gyro (a) and accelerometer (b) planned to be used to trigger the dropped foot implant.

Over the last 2 years, pilot clinical trials have been successfully completed in the UK and Holland, and the first commercially available devices will be available shortly through Finetech Medical.

The triggering mechanism is now being addressed as part of an ongoing funded project funded by the European Commission (EC). ${ }^{1}$ The intention is to replace the foot switch with an inertial unit mounted in the external power module. This will include accelerometers and a gyro (see Figure 2) being developed by ETB to detect leg movement. Intelligent signal processing being developed in Holland will relate this data to heel rise and subsequently trigger the implant.

The concept of using accelerometers and gyros for monitoring human movement is still relatively new; most systems have used sensors not designed for this type of application, but for high-volume, low-cost applications such as automotive. However, automotive requirements are high ' $\mathrm{g}$ ' or high angular rate, and low cost. The human motion application requires low ' $\mathrm{g}$ ', low angular rate and must be very small so as not to affect or restrict movement. ETB noted this niche market and developed a 3-axis accelerometer and a gyro specifically for this application. Prototypes should be available in 2004 and integrated into the first systems by 2005 .

Future developments are planned to extend the capability of functional electrical stimulator (FES) systems to assist movement in other limbs, particularly arms. ETB will be working with the clinical team at Salisbury Hospital, FTM and Salford University on an EC-funded 4-year research project (Healthy Aims) to develop these systems. ${ }^{2}$

There are a number of other areas where rapid technological advances in microsystems and nanotechnology have enabled new medical products to be realised. For example, cochlear implants can be implanted into children, as young as 1 year, to provide hearing, greatly enhancing their chance to lead a relatively normal life.

Blindness also affects a number of people, and currently there is no cure. Over the next 4 years, the Healthy Aims project will enable a team of scientists in Belgium, Germany, Austria and the UK to start developing a cure for blindness and improve the cochlear implant to overcome deafness. ${ }^{3}$

These are just a few examples of how technological advances have been applied, or are being applied, to produce new medical products. All of the systems described here incorporate a microsystem and often will use biomaterials and nanotechnology.

\section{Notes}

1 TUBA (Transceiver and Inertial Unit for Biomedical Application), IST-2001. A 3-year project under the Microsystems Programme.

2 Healthy Aims. Implantable microsensors and microsystems for ambulatory measurement and control in medical products. Integrated project IST-2002. A 4-year project under the Microsystems Programme.

3 Press release (Dec 2003) available from the author on request. 


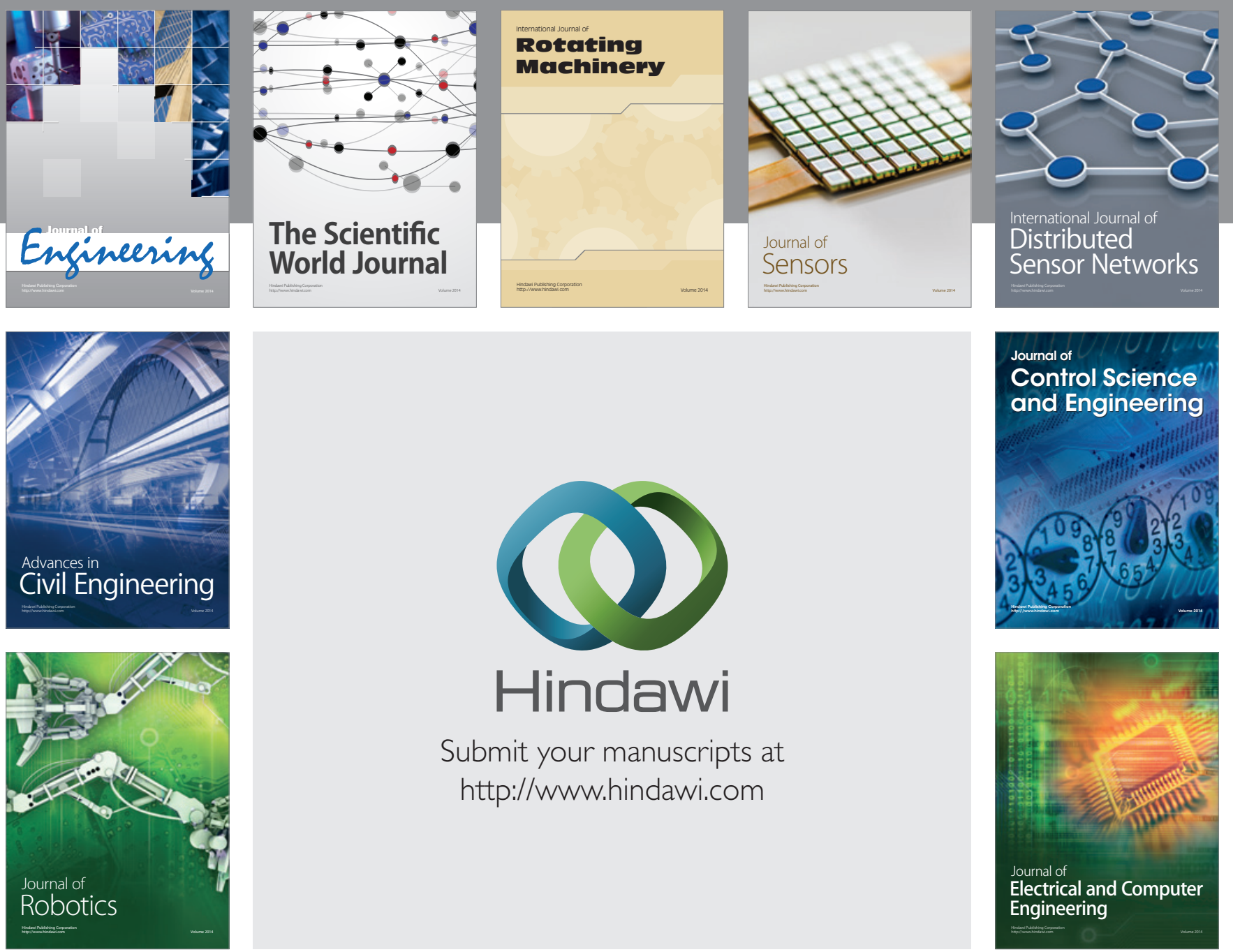

Submit your manuscripts at

http://www.hindawi.com
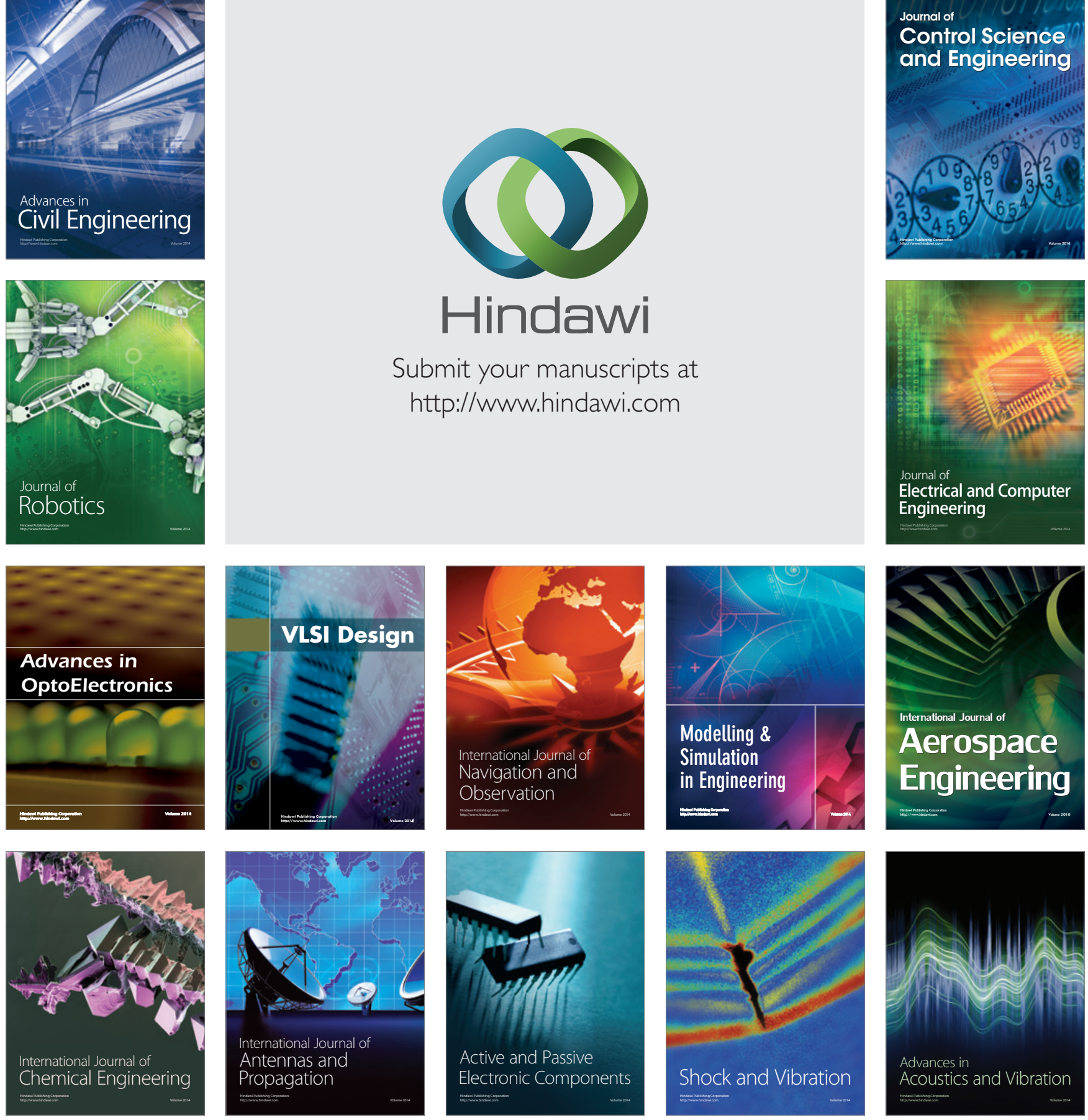